\title{
Un tecnólogo en medio de sus raíces
}

\author{
Guillermo Alfredo Vásquez Ortiz
}

\section{Resumen}

A lo largo de los años hemos entrado en una carrera loca por alcanzar los últimos avances tecnológicos para beneplácito de nuestra curiosidad, o por la utilidad de hacer más con menos. Hemos adoptado costumbres y formas de vida de otros países a través del uso de nuestro smartphone. No se ha considerado que en nuestro entorno y por las formas de vida de los pueblos indígenas existe también una manera de inundar de gigabytes a esta carretera de información llamada internet.

A través de una reciente experiencia de investigación de campo, se narra el encuentro entre las comunidades indígenas y la capacidad tecnológica, lo que permite mostrar al mundo las maravillas de reencontrarnos con nuestras raíces y con nuestra identidad. Se prueba que con ingenio y un poco de conocimiento de la web, podemos aportar historias que contribuyen a mantener vivas nuestras tradiciones y que además sirven para el disfrute de todos.

Palabras clave: Oaxaca, tecnología, festividades, rasgos culturales, identidad.

\section{A teChNologist IN THE MiddLe Of HIS ROOTS}

\begin{abstract}
Throughout the years we have adopted a crazy race to achieve the latest technological advances. For the sake of our curiosity or the convenience of doing more with less, we have adopted customs and lifestyles from other countries using our smartphone. We have not considered that in our own environment and in the lifestyle of indigenous people there are ways that would overflow with Gigabytes this highway of information called Internet.

Through a recent field research experience, indigenous communities and technology converge to show the world the importance and wonders of rediscovering our roots, our identity. We prove that creativity and knowledge can contribute to keep our traditions alive and also become enjoyable.
\end{abstract}

Keywords: Oaxaca, technology, festivities, cultural traits, identity. 


\section{Guillermo Alfredo Vásquez Ortiz}

Maestro en Mercadotecnia, Licenciado en Sistemas Computacionales y Diplomado en Práctica Educativa Innovadora con Tecnología Digital Integrada. Es catedrático en la Universidad La Salle. A lo largo de 24 años ha contribuido al desarrollo profesional de alumnos en universidades tecnológicas y de administración, ha impartido talleres, conferencias, foros y ha desarrollado múltiples investigaciones.

Es catedrático en diversas universidades como la Universidad del Pacífico de Chiapas, Universidad Regional del Sureste, Universidad Mesoamericana, Universidad Nacionalista México, Universidad Abierta y a Distancia de la Secretaría de Educación Pública, en la Maestría Administración de Negocios y actualmente en la Universidad La Salle Oaxaca en las carreras de Administración Turística, Ingeniería Industrial, Mercadotecnia Internacional, Sistemas Computacionales y la Ingeniería en Telemática, con las que ha contribuido al desarrollo de más de 68 proyectos que cubren aspectos sociales de Oaxaca.

Ha investigado sobre temas de tecnología en los organismos autónomos para mejorar su imagen institucional, implementando esquema de trabajo para automatizar procesos. El avance de esta investigación se publicó en "Las Tecnologías de la Información y Comunicaciones (TIC's) en los Organismos Autónomos Defensores de Derechos Humanos de la capital del Estado de Oaxaca" en 2010. Sus líneas de investigación son las tecnologías de la información, turismo, desarrollo local e imagen pública.

\section{Introducción}

Las siguientes líneas de texto van dedicadas a todos aquellos alumnos y usuarios de tecnologías que han abierto las puertas a nuevas formas de comunicarse y que encuentran en las redes sociales, los canales de video y el internet "el sentir de su mundo", con la invitación para arriesgarse a conocer lo que nuestros antepasados han dejado para estas generaciones y que sería interesante rescatar para beneplácito de todos.

A lo largo de los años vamos adoptando nuevas formas de relacionarnos, ya sea a través de videos virales que circulan en la web o cosas que nos gustan y se comparten en Facebook, Twitter e Instagram, permitiendo, además de entretenernos, y sin que sea esa la intención, la transculturación. Dicho término está definido por la Real Academia Española (2014) como la "recepción por un pueblo o grupo social de formas de cultura procedentes de otro, que sustituyen de un modo más o menos completo a las propias". Estas formas de cultura externas se dan por moda o tendencia, pero ¿no habrá manera de compartir, como espectador o protagonista, las formas de vida de nuestro pueblo, presentes y pasadas al mundo, mostrando lo valioso de nuestra tierra y sus habitantes? Si ya contamos con la creatividad, así como con algunos instrumentos tecnológicos como el smartphone, internet, redes sociales, y vivimos en espacios donde aún se observan las formas de vida de los pueblos originarios, isería fabuloso compartirlas! 
Mapa 1. Mixteca alta (Ortiz, 2019)
Quiero narrarles la aventura reciente que tuvimos al hacer investigación en la Mixteca alta, concretamente en las comunidades de San Andrés Sinaxtla, Santo Domingo Yanhuitlán y San Juan Bautista Coixtlahuaca del Estado de Oaxaca, México (ver mapa 1). Su objetivo era la recopilación de las festividades de desarrollo de vida humana (bautizos, bodas y funerales) y su difusión a través de talleres culturales. Dicha investigación fue lo que generó esta reflexión que ahora tiene en sus manos y cuya valía corresponde al trabajo interdisciplinario que hicimos en el equipo, cada uno, con sus aportes en diversas áreas del conocimiento.

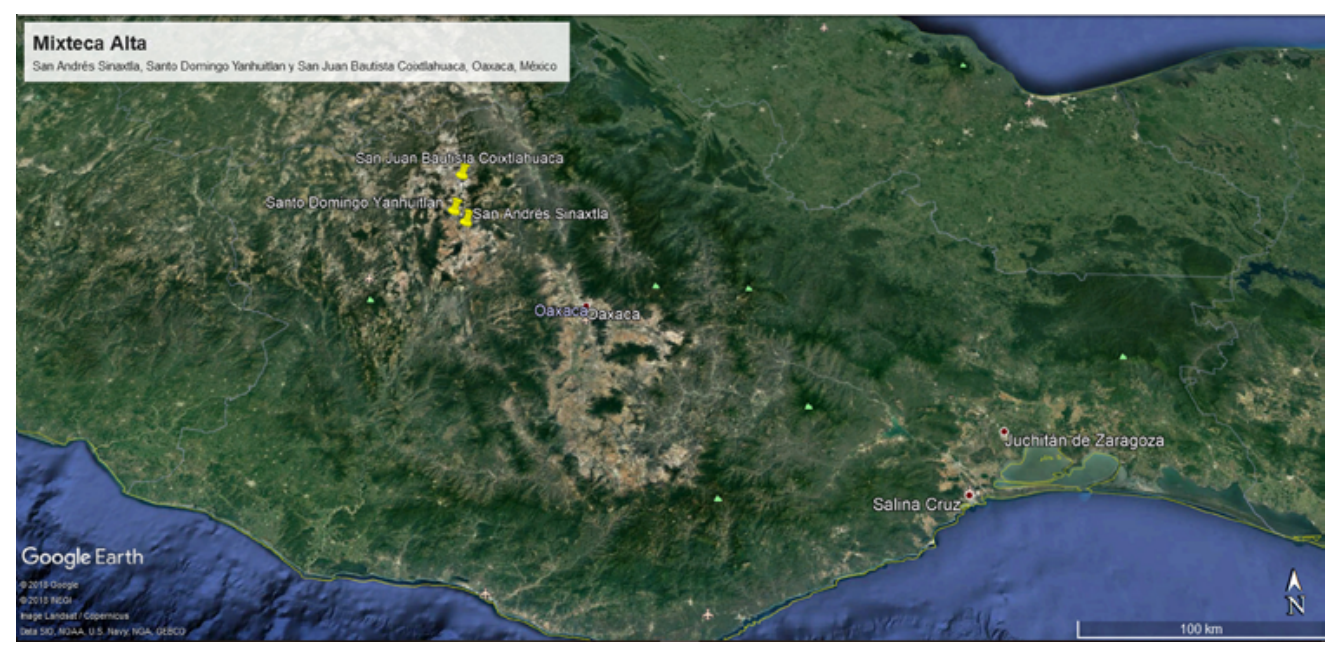

\section{En algún taller de tecnologías...}

Encontrándome en medio de los equipos de cómputo con los que habitualmente suelo trabajar, junto al rack polvoso que me ha acompañado a lo largo de estos últimos años, ese armazón donde colocamos equipos electrónicos e informáticos, recibí vía WhatsApp un mensaje del maestro Soto, un amigo de la Universidad La Salle. Me invitaba a realizar investigaciones en materia de cultura, turismo y festividades de una población cercana a la capital del estado. En ese momento vino a mi mente el recorrido hacia la Mixteca, lugar que Terraciano (2013) explica como una denominación histórica y cultural, en el sur de México a partir del nombre de sus habitantes más prominentes, los mixtecos. Sus límites abarcan, en dirección oeste-este, desde la frontera entre Guerrero y Oaxaca hasta el Valle de Oaxaca y, en dirección norte-sur, desde el sur de Puebla hasta el Océano Pacífico; y está dividida en cuatro regiones principales: Mixteca Alta, Mixteca Baja, Mixteca Costa y Valle de Oaxaca (ver mapa 2). El destino apuntaba hacia la Mixteca Alta del estado de Oaxaca y, para serles francos, no iba a dejar pasar la oportunidad de unirme al equipo y adicionar la perspectiva de la tecnología al equipo interdisciplinario conformado por la maestra Guadalupe, y los maestros Ramón, Eric y José Luis, especialistas en turismo y gastronomía. 


\section{Mapa 1}

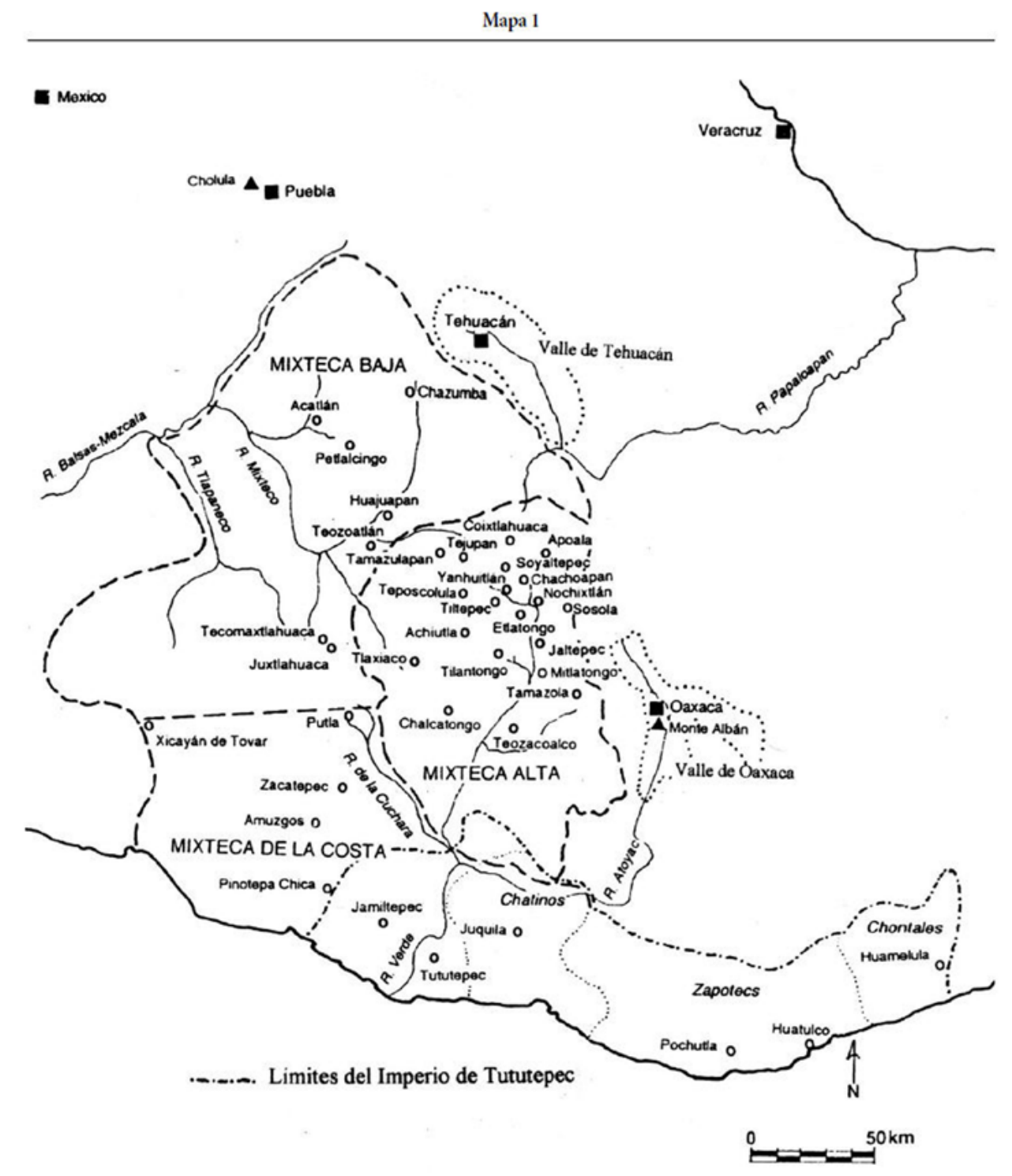

Una vez presentada la propuesta ante la Universidad la Salle Oaxaca y con la alegría de haber sido aceptada para llevarla a cabo, nos reunimos para repartir funciones y planear lo que realizaríamos. No obstante, nunca, nadie, consideró otros factores que encontraríamos a pocos minutos de la ciudad.

Nos embarcamos en busca de nuevos conocimientos, que así consideramos los que nos encontramos visitando esos lugares, mismos que nos compartieron personas con más de 50 años de vivir en esas regiones. La coincidencia y el tono de nostalgia en sus narrativas nos hacen pensar que los jóvenes y adultos de la región, quizá sin darse cuenta y a paso veloz, están dejando sus costumbres sepultadas junto con sus ancestros.
Mapa 2. Regiones de la Mixteca (Lind, 2018). 
Fotografía 1. Boda en Santo Domingo Yanhuitlán, Oaxaca, 1949 (Exposición del museo comunitario de Yahuitlán, 2017).
Ese es el caso de doña Crescencia (C. M. Cruz, comunicación personal, 3 de junio de 2017), quien nos comentó que recuerda, de cuando era joven, los preparativos de las bodas. Las personas se organizaban en la cocina, en equipos de trabajo comandados por el llamado Parangonero o Tonixano (leyenda hecha personaje, que más adelante se describe por la importancia que tiene en estas poblaciones). El Toxiano contaba en su ejército con cocineras, atoleras, molenderas, chocolateras y lavatrastes, para atender a las decenas de comensales que se congregan en la festividad. A esta congregación, que no recibía sueldo alguno y que ayudaba por invitación a quien lo requería, se le llama gueza, De ella se espera un "pago" futuro: que los beneficiados apoyen cuando se necesite.

De la señora Minerva (M. S. Cruz, comunicación personal, 30 de abril de 2017) escuchamos los relatos de su casamiento que se llevó a cabo de acuerdo con la tradición del acompañamiento. El novio y sus padres llevan una canasta de pan o algún presente, con la algarabía de comenzar la petición de la novia. Desde luego, cada familia debía llevar a su Tonixano, para que hablará en su nombre (ver fotografía 1).

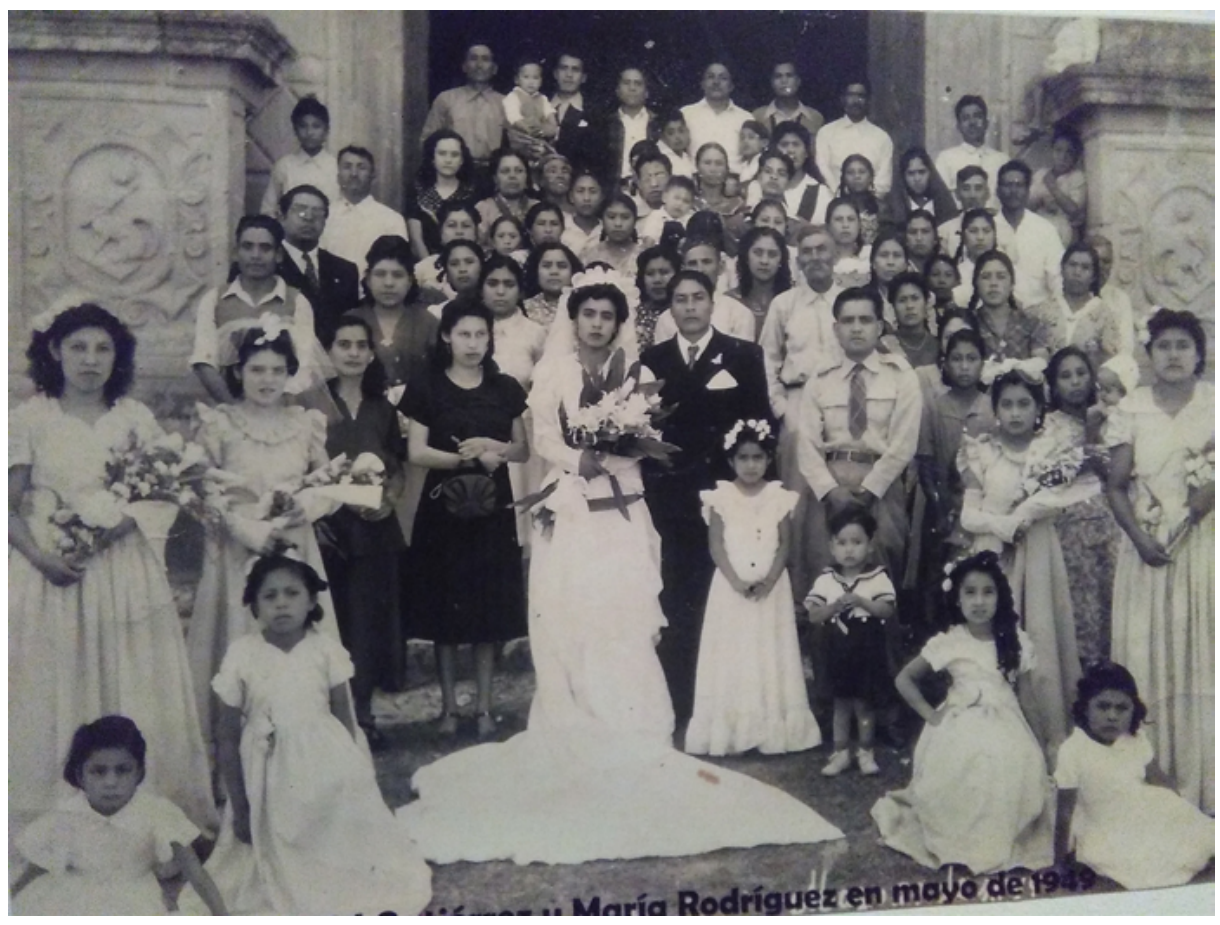

Éstas y otras personas de la región también nos compartieron información sobre las rutas que, con el paso de los años y de los habitantes, se configuraron para el acceso de los visitantes. La explicación, desde luego, venía acompañada de esa calidez natural de quien ve en el otro a un amigo que recibe, y al que le muestra con orgullo las bellezas ancestrales que vivieron y que ya pocas veces en estos días se observa. 
No sólo encontramos riqueza en sus palabras, también palpamos vestigios imponentes de culturas que nos narran, a cal y canto, sus formas de vida, confrontándonos con edificios de piedra, templos y hasta la preparación de comida, la cual es un manjar para los gustos más exigentes, preparada con alimentos regionales. Se trata de esa noción de cultura que Chávez (2014) nos explica como un conjunto de elementos materiales e inmateriales (lengua, ciencias, técnicas, instituciones, normas tradicionales, valores, símbolos, modelos de comportamiento socialmente transmitidos y asimilados, etcétera) que caracteriza a un determinado grupo humano en relación con los otros.

Obviamente, en muchos de estos lugares dejé de tener la "cobertura amplia" que garantiza la compañía contratada en mi smartphone, lo que me permitió estar sólo con los sonidos de la naturaleza y, en una que otra ocasión, con el de una campana lastimera, cuyo repique, comentaron los pobladores, anunciaba la muerte de una persona adulta del lugar, y que emitía un ritmo sereno, acompasado, triste, hacia la antesala de un funeral. Este sonido no es, sin embargo, el mismo que anuncia la muerte de un niño, a éste le llaman "agonías". (Insertar audio: agonías.mp3.)

\section{Y la leyenda toma forma}

Caminando por el sendero de los días y con las entrevistas realizadas a varios pobladores, cuyas arrugas nos mostraban el devenir de los años y sus ojos, las formas de vida de sus antepasados, entendimos el códice mixteco Vindobonensis (Anders, 1922), que narra el inicio de la luz. Cuando salió el sol por el oriente, llegaron hombres de descomunal tamaño y los seres sagrados (ñuhus) volvieron a entrar la tierra. En seguida se formaron los días, se asentó la muerte y su culto, se trazó el camino de las aguas. A esos seres se les conoció como gentiles, seres que dejaron huella en los mixtecos de esa época, y cuyos aportes perviven hasta nuestros días, salpicando de virtudes y enseñanzas a personas que transforman sus vivencias y contribuyen al fortalecimiento de sus raíces. Se trata de formas de vida que se niegan a morir y que ayudan de forma desinteresada a la sana convivencia de sus pobladores, personas enigmáticas que construyen las sociedades y la paz de los pueblos.

Nuestra sorpresa fue que, aún en estos días, se puede ver en algunas zonas a los encargados de transmitir el conocimiento y la cosmovisión mixteca, a los Parangoneros o Tonixanos. Sus raíces en la lengua mixteca están en toho, que significa noble, digno, señor, elegante, y nisanu que refiere a lo antiguo. Los términos juntos denotan a una persona que observa la tradición y que es sabio (Stephanie Wood, 2012).

Su representación en nuestros días existe en la conservación de enseñanzas, como nos lo dejó ver en una entrevista el señor Pastor (comunicación personal, 3 de junio de 2017). Él es Tonixano desde hace 15 años y fue nombrado por otro Tonixano. Su antecesor vio en el señor Pastor a un hombre con 
Fotografía 2. En el centro Sr. Pastor Román Bazán, Tonixano desde hace 15 años. conocimientos de la población, regido por la moral y buenas costumbres (ver fotografía 2). El Tonixano actual nos explicó, entre otras cosas, la participación que tiene, ya en menor medida, de "la cerrada de palabra": cuando se llega al término la petición de la novia. Así, su papel era primordial para la celebración de la boda, o para el fallecimiento de una persona, con su rol en los avisos trágicos a las autoridades e iglesia del municipio; también es él quien dice las palabras de resignación para despedir al difunto, hablando en representación de la familia y amistades.

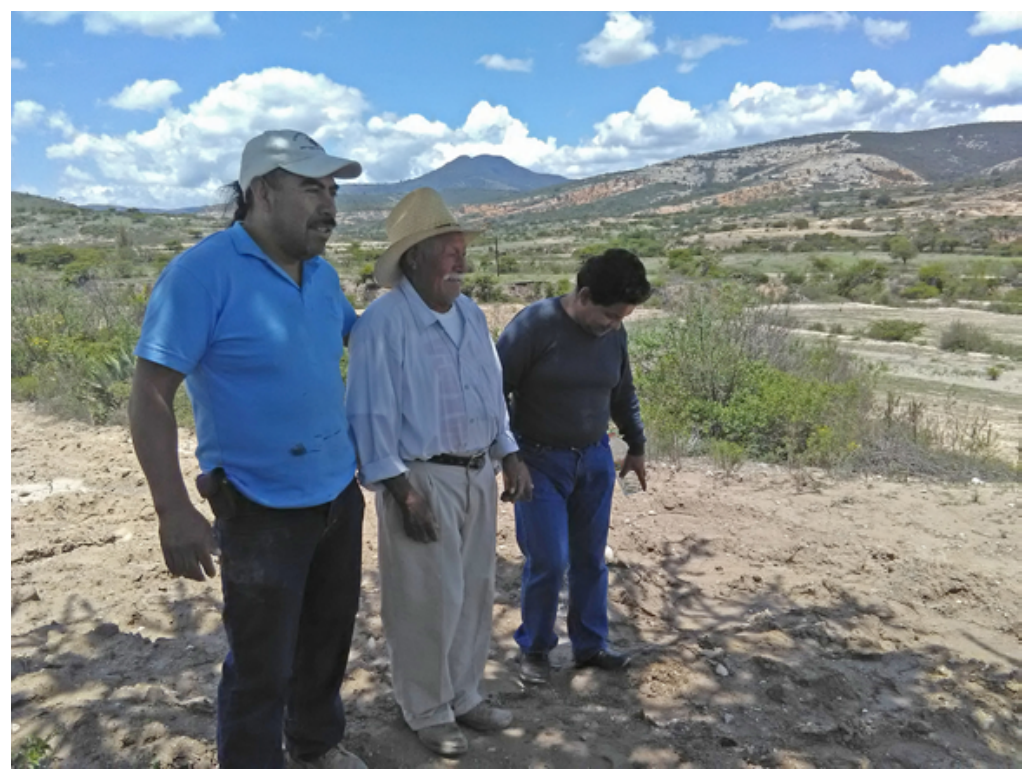

Del mismo modo, conversamos con el Tonixano Constantino Onofre, con 27 años de experiencia. Él nos narró que antes se acompañaba a pedir a la novia y se esperaba un plazo de dos o tres meses, para ver si los padres y la novia estaban de acuerdo. Después se arreglaba el casamiento civil y se ponía el plazo para la boda eclesiástica, momento en donde se pasaba al ofrecimiento y a la bendición de los papás del novio. Ahí el Tonixano encontraba las palabras sabias y adecuadas sobre la responsabilidad, obligaciones y derechos que obtendrían los novios.

De esta manera, durante algunos días más, los profesores y alumnos involucrados en la investigación vivimos en carne propia lo que los libros describen, como si se hubiera detenido el tiempo para contemplar el relato que las personas mayores nos proporcionaban sobre el pasado. Fue un encuentro impactante y cara a cara con nuestra identidad. Al encontrar las raíces de nuestras familias en esas regiones, los alumnos que nos acompañaron, como Viniza, Adriana, Efrén, Jesica y Eleuterio, y yo pensamos que debíamos compartir estas experiencias. Entonces, de manera natural, empleando la tecnología de nuestros smartphones e internet, presurosos tomamos fotografías y videos. Algunas de las entrevistas se pueden ver aquí, para que los demás, como nosotros, disfrute de los avances de la investigación. 
Además, fuimos más allá de encontrar documentos que narraron lo ocurrido hace años, sentimos las leyendas en la voz de los hijos de esos ancestros que un día se aparecieron en esas tierras, le encontramos sabor a las costumbres de esa región, a través de guisos como la barbacoa de borrego que se servía en los bautismos, o la particular forma de hacer la vitualla, platillo que por su color y sabor representa el momento de tristeza de un funeral. Todos estos momentos que los compartimos a través de éste video, en donde la Sra. Josefina López Pérez usa la preparación que hace ya 30 años no se realizaba en las manos expertas de la cocinera.

Lo maravilloso de esto es que permitió una reflexión de todos los que nos encontrábamos descubriendo un mundo rico en templos, en comida, en tradiciones. Tanto los maestros como los estudiantes aportamos ideas de cómo evitar que mueran estas formas de vida, desde talleres, cursos, tesis, hasta la creación de un portal multimedia donde se pudiera difundir todo aquello que motive interés.

\section{Conclusión}

Si bien es cierto que existen algunos portales que muestran la vida de los pueblos indígenas, como el sitio web Cartografía Lacustre, queremos aportar uno donde se estén recuperando las tradiciones de una zona como la Mixteca, a través de las delicias culinarias que antes se realizaban. De esta manera, se hizo el esfuerzo de llevarlo a los alumnos de gastronomía y turismo para su reproducción y difusión.

Este encuentro entre las formas de vida en los pueblos indígenas y de la tecnología en los medios de comunicación para traerlas al presente, para hacerlas visibles, es algo que debe compartirse entre todos: profesores, alumnos y cibernautas, para contar, desde nuestra perspectiva, la historia de nuestros abuelos y no sólo lo que los libros de texto narran. Para ello se emplea internet y una cámara, que reduce las distancias a un sólo "clic". Estamos convencidos de que combinar el interés con la tecnología da resultados extraordinarios y que no deja de lado los procesos que según Cobo (2016) deben existir para generar conocimiento significativo en los estudiantes, como son: convivencia escolar, autoestima y confianza, asistencia y retención escolar, estímulo, acompañamiento, reforzamiento, motivación, pertenencia, comunidad, etcétera.

Estimada y estimado estudiante: ¿te imaginas poder llevar relatos a la web de esta naturaleza, combinando toda la parafernalia multimedia, entrevistas a los pobladores y las manifestaciones culturales de bautismos, bodas o despedidas a seres queridos a través de los funerales? Sin duda ayudarías a enriquecer la cantidad de gigas que se observan cada día en internet.

En ese sentido, te compartimos en el canal de Youtube, Un tecnólogo en medio de sus raíces lo que nos sorprendió al escudriñar las maravillas de nuestra tierra, esperando que sea de tu agrado. Nos vemos en la web. 


\section{Referencias}

* Anders, F. M. (1922). Códice Vindobonensis. Origen e historia de los reyes mixtecos. México: Fondo de Cultura Económica (FCE).

* Chávez, J. (2014). Diversidad cultural y ecoturismo. México: Editorial Trillas.

* Cobo, C. (2016). La Innovación Pendiente. Reflexiones (y Provocaciones) sobre educación, tecnología y conocimiento. Montevideo: Colección Fundación Ceibal/ Debate.

* Exposición del museo comunitario de Yahuitlán. (Abril de 2017). Santo Domingo Yanhuitlán, Oaxaca, México.

* Lind, M. (2008). Arqueología de la Mixteca. Desacatos, (27), 13-32. Recuperado de: http://www.scielo.org.mx/scielo.php? script=sci arttext\&pid=\$1607-050X200 8000200002\&lng=es\&tIng=es.

- Ortiz, G. V. (2019). Mapa 1 Mixteca Alta. Recuperado de: https://www.google.com. mx/maps.

* Real Academia Española. (2014). Diccionario de la lengua española (23.a ed.). Recuperado de: https://dle.rae.es/?id=algYBxO .

- Stephanie Wood, A. M. (2012). Introduction: Collective Memory and Mesoamerican Systems of Remembrance. En Amos Megged y Stephanie Wood (Eds.), Mesoamerican Memory: Enduring Systems of Remembrance (3-14). Norman: University of Oklahoma Press.

- Terraciano, K. (2013). Los mixtecos de la Oaxaca colonial. La historia nudzahui del sig/o XVI al XVIII (Pedro Escalante Gonzalbo, Trad.). México: FCE. Recuperado de: http:// www.fondodeculturaeconomica.com/subdirectorios site/libros electronicos/ desde la imprenta/006227R/files/terraciano los\%20mixtecos\%20de\%20la\%20 oaxaca.pdf.

\section{Cómo citar este artículo}

* Vásquez Ortiz, Guillermo Alfredo (2019). Un tecnólogo en medio de sus raíces. Revista Digital Universitaria (RDU). Vol. 20, núm. 4 julio-agosto. Dol:http://doi. org/10.22201/codeic.16076079e.2019.v20n4.a6.

Recepción: 04/09/2017 Aprobación: 17/05/2019. 